\title{
Formulation and Evaluation of Chronomodulated Drug Delivery of Montelukast Sodium
}

\author{
Madhuri Asiniparthi*, Anusha and Vaidehi Donti \\ Department of Pharmaceutics, Gyana jyothi College Of Pharmacy, India
}

Submission: February 01, 2016; Published: February 20, 2016

*Corresponding author: Madhuri Asiniparthi, Department of Pharmaceutics, Gyana jyothi College Of Pharmacy, Hyderabad-500039, India, Tel: 0939314418; 0939314412; Email: gyana.jyothi@gmail.com

\begin{abstract}
An oral press coated tablet containing Montelukast sodium was formulated with an outer barrier layer of different compositions of hydrophobic polymer eythyl cellulose and hydrophilic polymer hydroxyl propyl methyl cellulose. This press coated tablet was prepared by using direct compression and wet granulation methods in order to achieve the predetermined lag time.

Keywords: Pulsatile drug delivery systems (PDDS); Nocturnal asthma(NA); Chronopharmacology; Circadium Rhythms; Burst release; Lag time

Abbreviations: EC: Ethyl Cellulose; HPMC: Hydroxy Propyl Methyl Cellulose; FTIR: Fourier Transform Infra Red; SLS: Sodium Lauryl Sulphate; IR: Immediate Release; ChrDDS: Chrono Modulated Drug Delivery Systems; PCT: Press Coated Tablets
\end{abstract}

\section{Introduction}

Controlled drug delivery systems [1] have acquired a centre stage in the area of pharmaceutical R \&D sector. Such systems offer temporal \&/or spatial control over the release of drug and grant a new lease of life to a drug molecule in terms of controlled drug delivery systems for obvious advantages of oral route of drug administration. These dosage forms offer many advantages, such as nearly constant drug level at the site of action, prevention of peak-valley fluctuation, reduction in dose of drug, reduced dosage frequency, avoidance of side effects and improved patient compliance. In such systems the drug release commences as soon as the dosage form is administered as in the case of conventional dosage forms. However, there are certain conditions, which demand release of drug after a lag time. Such a release pattern is known as pulsatile release [2-5]. The diseases currently targeted for chronopharmaceutical formulations are those for which there are enough scientific backgrounds to justify ChrDDS compared to the conventional drug administration approach. These include asthma, arthritis, duodenal ulcer, cancer, diabetes, cardiovascular diseases, hypercholesterolemia, ulcer and neurological diseases [6,7].

If the organization in time of living system including man is borne in mind, it is easy to conceive that not only must the right amount of the right substance be at right place but also this must occur at the right time. In the last decade numerous studies in animals as well as clinical studies have provided convincing evidence, that the pharmacokinetics \&/or the drug effects -side effects can be modified by the circadian time \&/or the timing of drug application within $24 \mathrm{hrs}$ of a day [8]. A pulsatile drug delivery system that can be administered at night (before sleep) but that release drug in early morning would be a promising chronopharmaceutic system. Drug targeting to colon [9] would prove useful where intentional delayed drug absorption is desired from therapeutic point of view in the treatment of disease that have peak symptoms in the early morning such as nocturnal asthma, angina, arthritis.

Circadian rhythms are self-sustaining, endogenous oscillation, exhibiting periodicities of about one day or 24 hours. Normally, circadian rhythms are synchronized according to the body's pacemaker clock, located in the suprachiasmic nucleus of the hypothalamus [8]. Asthma is a chronic inflammatory disease of the airways, characterized by hyper responsiveness to a variety of stimuli. The role of circadian rhythms in the pathogenesis and treatment of asthma indicates that airway resistance increases progressively at night in asthmatic patients. Circadian changes are seen in normal lung function, which 
reaches a low point in the early morning hours. The worsening of asthma at night commonly referred to as nocturnal asthma (NA) [10]. A drug delivery system administered at bedtime but releasing drug during morning hours would be ideal in this case. Nocturnal asthma is a variable exacerbation of the underlying asthma condition associated with increases in symptoms, need for medication, airway responsiveness, and/or worsening of lung function. Generally, a reduction in peak flow or forced expiratory volume in one second (FEV1) of at least $20 \%$ is implicit in this definition. Lung function (e.g., peak expiratory flow rate or FEV1) is usually highest at $4 \mathrm{PM}$ and lowest at $4 \mathrm{AM}$ the latter time is generally when asthma symptoms are most prevalent. . Consequently, the administration of a drug formulated in such a delivery system, i.e. taken at bedtime with a programmed start of drug release in early morning hours, could offer a more effective therapy than a typical controlled release drug delivery system, provided that the most appropriate drugs are administrated [11].

Pharmaceutical coatings [12] are an essential tool to achieve the desired formulation of pharmaceutical dosage forms. Coating techniques mostly used in pharmaceutical industry are aqueous or organic coating, which present some disadvantages: time consuming, stability for heat labile and hydrolysis of degradable drug and polluted environment problem. Thereby, non-solvent coating is introduced as alternative coating technique to overcome these disadvantages.

For the time controlled release system from compressioncoated tablets, the amount of the outer shell is a key factor for controlling the lag time. Higher amount of the outer coating added would prolong the lag time of drug release [13].

The aim of the present investigation was to develop and evaluate an alternative, simple, orally applicable one pulse drug delivery system based on a press-coated tablet preparation. The PCT investigated in the current study consisting of a rapidly disintegrating core tablet presscoated by a barrier layer consisting of varying concentrations of Hydroxy propyl methyl cellulose (HPMC) and Ethylcellulose (EC). HPMC is a disintegrant and had been used to cause rapid disintegration of tablets. The other component of the barrier layer, Ethylcellulose (EC) is a well-known water-insoluble polymer that has long been used as a rate-controlling membrane in medication dosage forms to regulate drug release. Although EC has also been added in tablet formulations to act as a retarding material, few papers have focused on the use of EC as a directly compressible excipient. It was postulated that when the barrier layer was exposed to dissolution media, the HPMC particles swell and erode [14], a process which was retarded to varying degrees depending upon the quantity of EC present, demonstrating that manipulation of both components controls the erosion rate.

\section{Materials and Methods}

Table 1: List of chemicals

\begin{tabular}{|c|c|c|}
\hline S.No & Name of chemicals & Source \\
\hline 1. & $\begin{array}{c}\text { Drug } \\
\text { Montelukast Sodium }\end{array}$ & Gift sample obtained from the Madras Pharmaceuticals Ltd, Chennai \\
\hline & Polymer & Drugs India, Hyderabad \\
2. & HPMC K $\mathrm{M}_{4}$ & Drugs India, Hyderabad \\
3. & EC T10 & \\
\hline & Excipients & Drugs India, Hyderabad \\
4. & Lactose & Drugs India, Hyderabad \\
5. & Ac-di-sol & Kawarlal \& Co., Chennai \\
6. & Primojel & Ridesh Chemicals Pvt Ltd., Mumbai \\
7. & Crospovidone & Harish Chemicals Pvt Ltd., Ahmadabad \\
8. & Magnesium Stearate & abot Sunmar Pvt Ltd., Naddoor \\
9. & Colloidal sio & \\
\hline
\end{tabular}

\section{Preparation of core tablets by direct compression}

The ingredients depicted in the table except colloidal silicon dioxide and magnesium stearate were dry blended for 15 minutes followed by addition of quitted ingredients and dry blended for another 5 minutes. The mixed blend of drug and excipients was compressed using a single punch CADMACH punching machine to produce round tablets weighing $100 \mathrm{mg}$ with a diameter of $6 \mathrm{~mm}$. A minimum of 50 tablets were prepared for each batch. 
Table 2: Formulation of the core tablet.

\begin{tabular}{|c|c|c|c|c|c|c|c|c|c|}
\hline $\begin{array}{c}\text { Ingredients } \\
\text { (mg/100mg tab) }\end{array}$ & F1 & F2 & F3 & F4 & F5 & F6 & F7 & F8 & F9 \\
\hline Montelukast sodium & 10 & 10 & 10 & 10 & 10 & 10 & 10 & 10 & 10 \\
\hline Lactose monohydrate & 83.6 & 81.1 & 78.6 & 83.6 & 81.1 & 78.6 & 83.6 & 81.1 & 78.6 \\
\hline Ac-di-sol & 5 & 7.5 & 10 & - & - & - & - & - & - \\
\hline Primojel & _- & _- & _- & 5 & 7.5 & 10 & _- & - & - \\
\hline Polyplasdone XL10 & _- & - & - & - & - & _- & 5 & 7.5 & 10 \\
\hline Colloidal $\mathrm{SiO}_{2}$ & 0.4 & 0.4 & 0.4 & 0.4 & 0.4 & 0.4 & 0.4 & 0.4 & 0.41 \\
\hline Magnesium stearate & 1 & 1 & 1 & 1 & 1 & 1 & 1 & 1 & 1 \\
\hline
\end{tabular}

\section{Preparation of press-coated tablets}

The core tablets were press-coated with $400 \mathrm{mg}$ of prepared barrier blend as per the mentioned formulas from X1 to X5. $200 \mathrm{mg}$ of barrier layer material was weighed and transferred into a $13 \mathrm{~mm}$ die then the core tablet was placed manually at the center. The remaining $200 \mathrm{mg}$ of the barrier layer material was added into the die and compressed.

Table 3: Formulation of Barrier layer ( $400 \mathrm{mg}$ ) for press coated tablets.

\begin{tabular}{|l|c|c|c|c|c|}
\hline \multirow{2}{*}{ Ingredients } & \multicolumn{5}{|c|}{ Formulation (\%W/W) } \\
\cline { 2 - 6 } & $\mathbf{X 1}$ & $\mathbf{X 2}$ & $\mathbf{X 3}$ & $\mathbf{X 4}$ & $\mathbf{X 5}$ \\
\hline EC T 10 & 100 & 87.5 & 0 & 50 & 25 \\
\hline HPMC $_{100} \mathrm{M}$ & 0 & 12.5 & 100 & 50 & 75 \\
\hline
\end{tabular}

\section{In vitro drug release study of core tablets}

The in vitro release pattern of core tablets was studied as per method given by Chaudhari SP [15] Release pattern was studied visually by taking images of the core tablets in a petri plate containing dissolution medium at the specific time intervals $5 \mathrm{sec}, 10 \mathrm{sec}, 15 \mathrm{sec}$. Also the sample was analyzed at $342 \mathrm{~nm}$ using a UV spectrophotometer.

\section{In vitro drug release study of press-coated tablets}

In-vitro dissolution studies of press coated tablets were performed at $37 \pm 0.5{ }^{\circ} \mathrm{C}$ using $0.5 \% \mathrm{w} / \mathrm{v}$ aqueous solution sodium lauryl sulfate in USP II paddle method at $50 \mathrm{rpm} .5 \mathrm{ml}$ of filtered aliquot was manually withdrawn at pre-determined time intervals and replaced with $5 \mathrm{ml}$ of fresh $0.5 \%$ sodium lauryl sulfate solution maintained at the same temperature. The samples were analysed at $342 \mathrm{~nm}$ using a UV spectrophotometer. The lag time and percentage release was determined of the each formulation.

\section{Results and Discussion}

\section{Design of Pulsatile release tablet}

The pulsatile drug delivery system consisted of inner core tablet containing drug reservoir and outer coating layer with combination of water insoluble polymer Ethylcellulose and water soluble polymer HPMC. Ethyl cellulose was chosen because of its swelling and rupturable behavior. HPMC was chosen because of its eroding behavior.

\section{In Vitro dissolution of core tablets}

The core tablet shows $73.97 \%$ of drug release within 9 minutes upon contact with dissolution medium, core tablet get erode and release the drug as given in Figure 1.

\section{Analytical methods}

From the standard stock solution $(1000 \mu \mathrm{g} / \mathrm{ml})$, appropriate aliquot were transferred to series of $10 \mathrm{ml}$ volumetric flasks and made upto $10 \mathrm{ml}$ with desired solvents so as to get concentration of $5,10,15,20 \ldots$ or $2,4,6,8 \ldots \mu \mathrm{g} / \mathrm{ml}$. the absorbance of the solution were measured at $342 \mathrm{~nm}$ for Montelukast sodium. This procedure was performed in triplicate to validate calibration curve. A calibration curve was plotted.

Table 4: - Linearity values for Montelukast sodium

\begin{tabular}{|c|c|c|}
\hline S.No & Concentration $(\boldsymbol{\mu g} / \mathbf{m l})$ & Absorbance $(\mathbf{n m})$ \\
\hline 1 & 0 & 0 \\
\hline 2 & 2 & 0.1706 \\
\hline 3 & 4 & 0.3446 \\
\hline 4 & 6 & 0.4886 \\
\hline 5 & 8 & 0.6423 \\
\hline 6 & 10 & 0.8206 \\
\hline
\end{tabular}




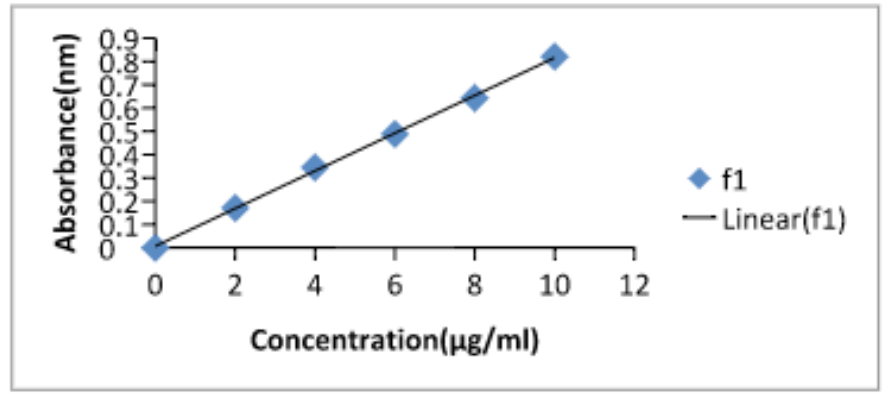

Figure 1: Standard plot of Montelukast sodium in $0.5 \%$ SLS at $\lambda \max 342 \mathrm{~nm}$.

\section{Compatibility Analysis}

\section{Fourier transform infra-red spectroscopy}

FT-IR spectroscopy was employed to ascertain the compatibility of drugs with polymers. The individual drug and final formulation were scanned. Both the spectra were compared for confirmation of common peaks. Montelukast sodium with

Table 5: FTIR spectra data of Montelukast sodium and final formulation polymers showed no significant variation in height, intensity and position of peaks, suggesting that drug and excipients were compatible. There is no interaction between drug and polymer. Hence, it can be concluded that the drug is in Free State and can release easily from the formulation the spectra are reported in the Table no.6.3 and Figure 6.2-6.3.

\begin{tabular}{|c|c|c|c|c|}
\hline & N-H & C-H & C=0 & C-Cl \\
\hline MONTEL pure drug & $3442 \mathrm{~cm}^{-1}$ & $2929 \mathrm{~cm}^{-1}$ & $1715 \mathrm{~cm}^{-1}$ & $800 \mathrm{~cm}^{-1}$ \\
\hline Final formulation & $3446 \mathrm{~cm}^{-1}$ & $2935 \mathrm{~cm}^{-1}$ & $1720 \mathrm{~cm}^{-1}$ & $746 \mathrm{~cm}^{-1}$ \\
\hline
\end{tabular}

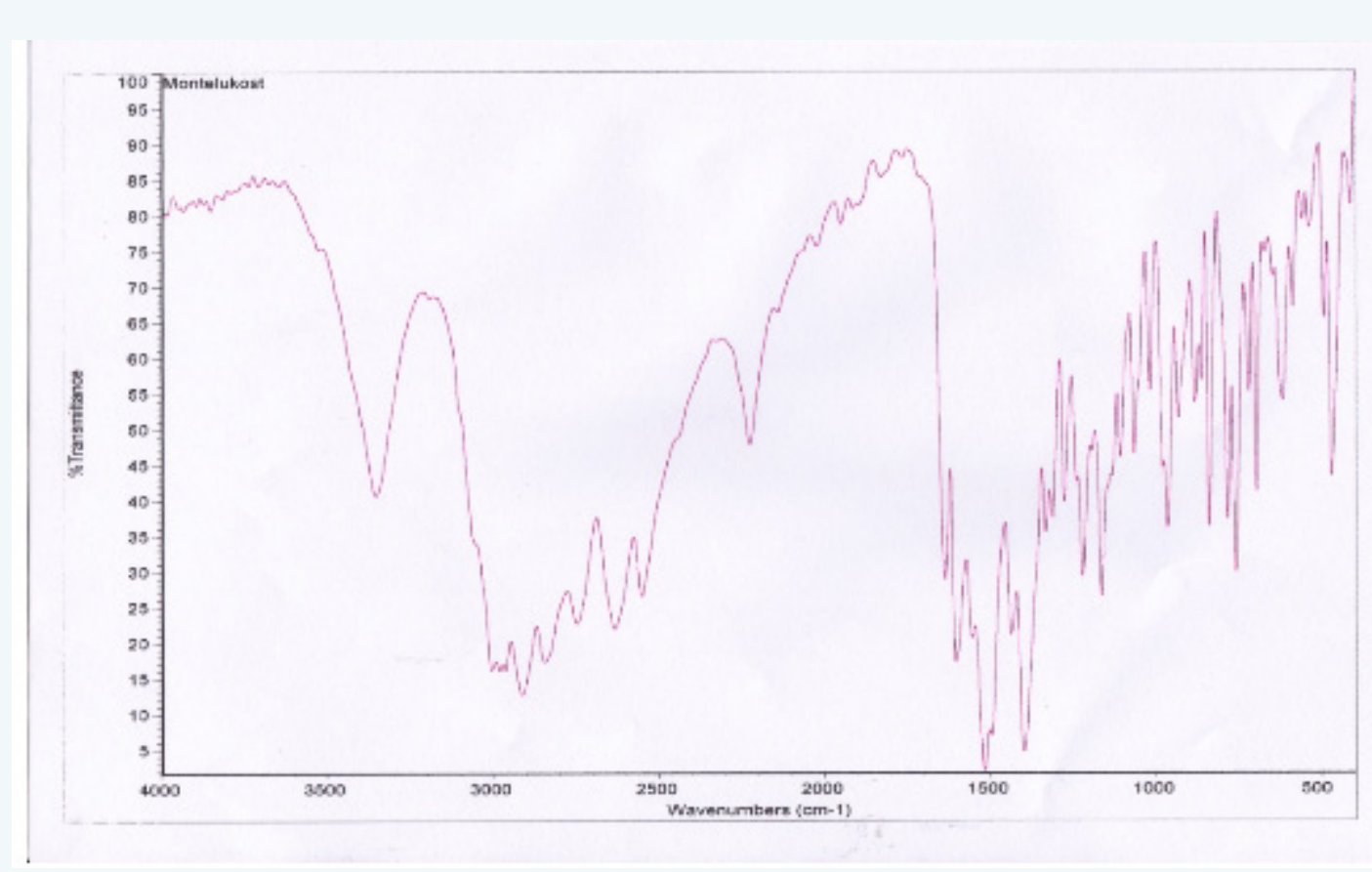

Figure 2: FTIR spectrum of Montelukast pure drug. 


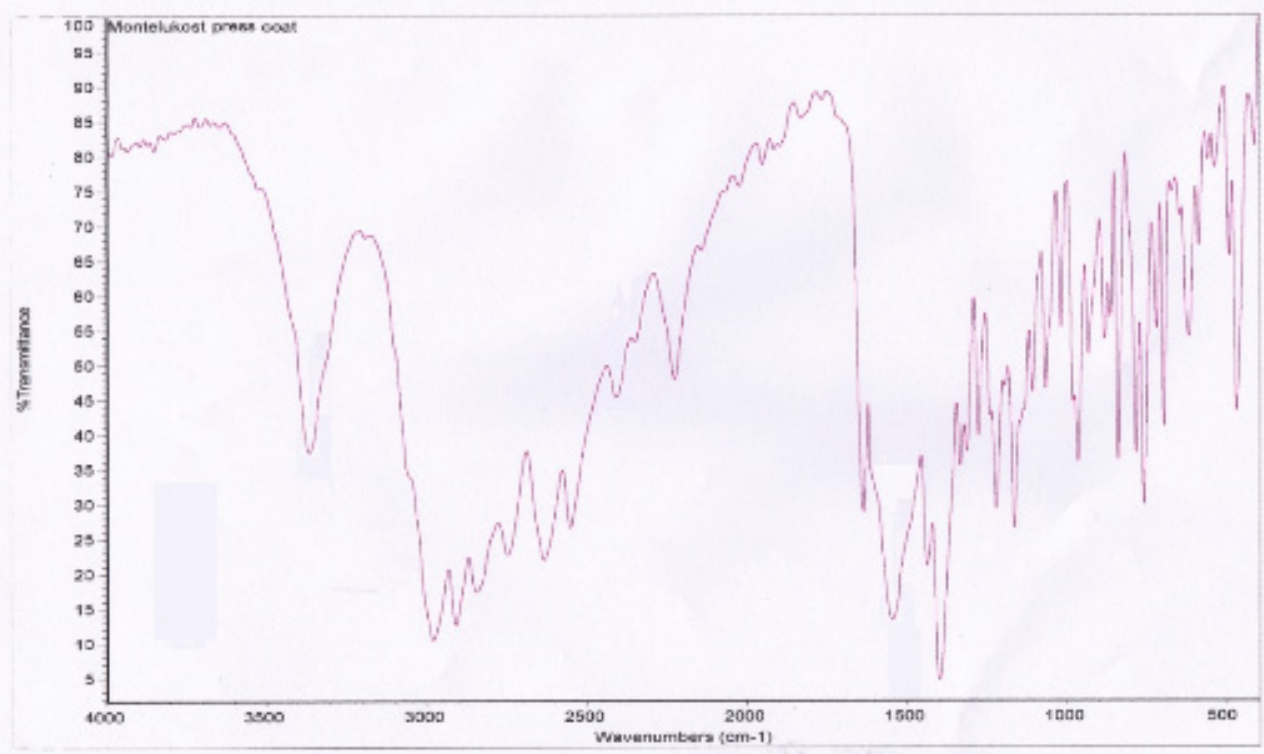

Figure 3: FTIR spectrum of Final formulation.

Table 6: Dissolution studies for core tablet.

\begin{tabular}{|c|c|c|c|c|c|c|c|c|c|}
\hline & \multicolumn{9}{|c|}{ Cumulative \% drug release } \\
\hline Time (mins) & F1 & F2 & F3 & F4 & F5 & F6 & F7 & F8 & F9 \\
\hline 0 & 0 & 0 & 0 & 0 & 0 & 0 & 0 & 0 & 0 \\
\hline 3 & 31.27 & 34.22 & 29.32 & 30.34 & 32.43 & 35.55 & 37.33 & 39.65 & 33.26 \\
\hline 6 & 54.31 & 49.34 & 52.64 & 54.51 & 47.54 & 56.41 & 53.24 & 60.24 & 50.75 \\
\hline 9 & 65.67 & 56.55 & 65.09 & 63.11 & 59.43 & 63.62 & 66.68 & 73.97 & 62.75 \\
\hline 12 & 79.33 & 68.05 & 81.87 & 87.13 & 75.32 & 70.22 & 78.65 & 87.47 & 73.97 \\
\hline 15 & 88.11 & 79.66 & 92.68 & 93.14 & 91.25 & 89.72 & 94.43 & 98.96 & 86.26 \\
\hline
\end{tabular}

Table 7: Correlation Coefficient $r^{2}$ Values of immediate release core tablets of Montelukast sodium formulated employing different Super disintegrants as per Zero Order and First Order Kinetics.

\begin{tabular}{|c|c|c|c|c|}
\hline \multirow{2}{*}{ Formulation code } & \multicolumn{4}{|c|}{ Correlation coefficient $\left(\mathbf{r}^{2}\right)$} \\
\cline { 2 - 5 } & Zero order & First order & Higuchi & Peppas \\
\hline F1 & 0.935 & 0.952 & 0.986 & 0.883 \\
\hline F2 & 0.975 & 0.936 & 0.983 & 0.882 \\
\hline F3 & 0.943 & 0.950 & 0.983 & 0.889 \\
\hline F4 & 0.832 & 0.952 & 0.897 & 0.861 \\
\hline F5 & 0.957 & 0.980 & 0.976 & 0.865 \\
\hline F6 & 0.841 & 0.969 & 0.954 & 0.839 \\
\hline F7 & 0.963 & 0.987 & 0.871 & 0.816 \\
\hline F8 & 0.856 & 0.999 & 0.964 & 0.869 \\
\hline
\end{tabular}




\section{Global Journal of Pharmacy \& Pharmaceutical Sciences}

Table 8: First order linear regression equations, rate of drug release of different formulations

\begin{tabular}{|c|c|c|}
\hline \multirow{2}{*}{ Formulation Code } & Zero order & First order \\
\cline { 2 - 3 } & $\mathbf{K}_{\mathbf{0}}$ & 0.037 \\
\hline F1 & 1.08 & 0.032 \\
\hline F2 & 1.38 & 0.033 \\
\hline F3 & 1.41 & 0.038 \\
\hline F4 & 1.30 & 0.062 \\
\hline F5 & 1.36 & 0.043 \\
\hline F6 & 1.13 & 0.088 \\
\hline F7 & 1.41 & 0.138 \\
\hline F8 & 1.90 & 0.095 \\
\hline F9 & & \\
\hline
\end{tabular}

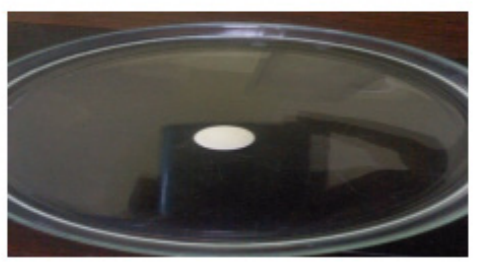

5(a): $0 \mathrm{sec}$

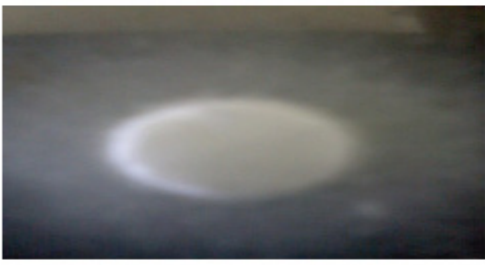

5(c) $10 \mathrm{sec}$

Figure 4: Wetting time of the optimized core tablet.

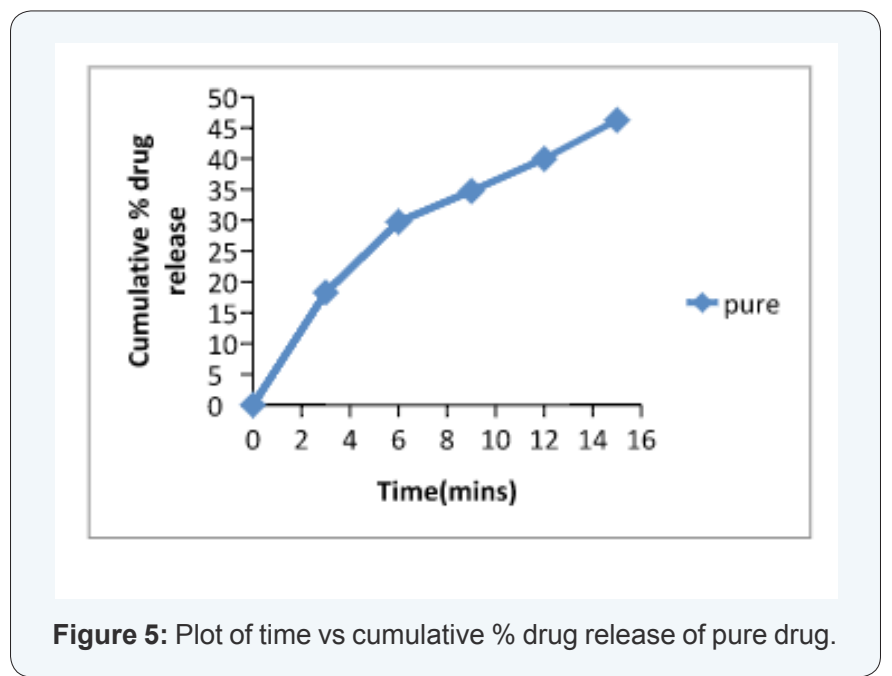

Figure 5: Plot of time vs cumulative \% drug release of pure drug.

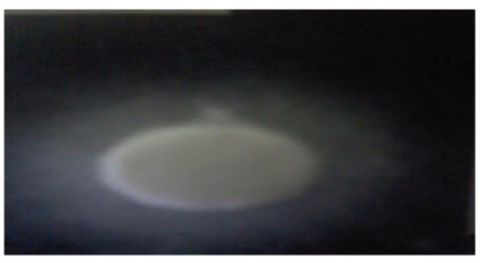

5(b): 5 sec

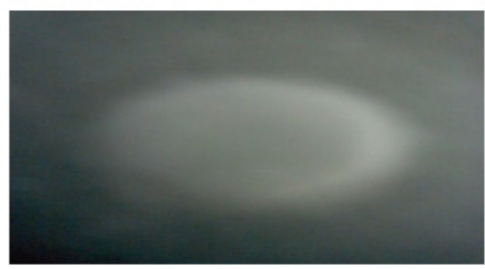

5(d): 15 sec

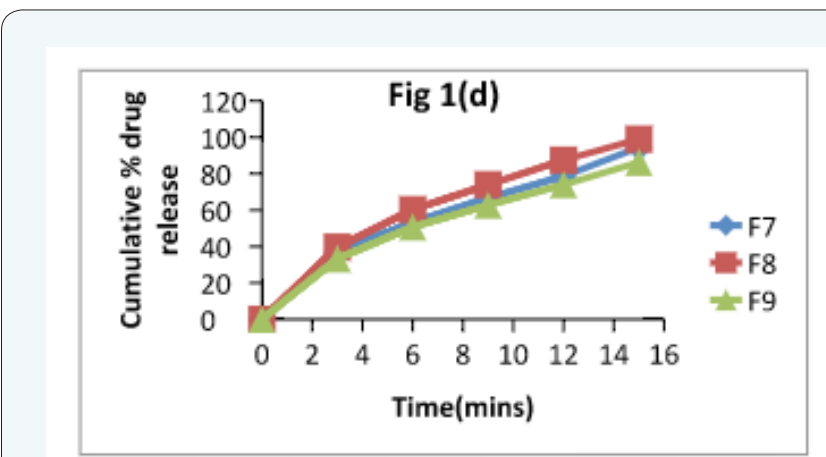

Figure 6: Plot of time vs cumulative \% drug release of F7, F8, F9 formulations.

Based on the drug release within the required time period F8 was optimized and further formulated for press coating. 


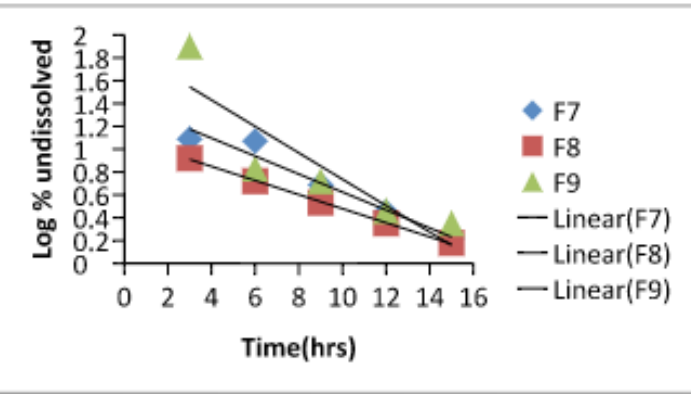

Figure 7: Plot of time vs log \% undissolved of F7, F8, F9 formulations.

Table 9: Dissolution profile of Press coated tablets.

\begin{tabular}{|c|c|c|c|c|c|}
\hline \multirow{2}{*}{ Time(hrs) } & \multicolumn{5}{|c|}{ Cumulative \% drug release } \\
\hline & $\mathbf{X 1}$ & $\mathrm{X} 2$ & X3 & $\mathrm{X} 4$ & $\mathbf{X 5}$ \\
\hline 0.5 & - & - & - & - & _- \\
\hline 1 & - & - & 2.74 & - & - \\
\hline 1.5 & - & - & 79.7 & _- & - \\
\hline 2 & - & - & 82.3 & 4.8 & - \\
\hline 2.5 & - & - & 84.1 & 42.6 & - \\
\hline 3 & - & - & 86.1 & 71.6 & 3.58 \\
\hline 3.5 & - & - & 86.8 & 75.3 & 47.11 \\
\hline 4 & - & 6.33 & 89.3 & 78.4 & 68.86 \\
\hline 4.5 & - & 83.57 & 91.1 & 80.5 & 74.9 \\
\hline 5 & 4.09 & 98.63 & 92.4 & 82.6 & 79.5 \\
\hline 5.5 & 36.44 & 98.95 & 92.6 & 86.4 & 85.3 \\
\hline 6 & 76.17 & - & 93.9 & 89.1 & 90.7 \\
\hline
\end{tabular}

Table 10: Correlation coefficient ( $r 2$ ) values in the analysis of release data of Montelukast sodium Press coated tablets as per various kinetic models.

\begin{tabular}{|c|c|c|c|c|c|}
\hline \multirow{2}{*}{ Formulation Code } & \multicolumn{5}{|c|}{ Correlation coefficient ( $\mathbf{r}^{2}$ ) } \\
\cline { 2 - 6 } & Zero order & First order & Higuchi & Peppes & n value of peppas \\
\hline F1 & 0.870 & 0.782 & 0.684 & 0.856 & 0.641 \\
\hline F2 & 0.839 & 0.991 & 0.626 & 0.740 & 0.740 \\
\hline F3 & 0.949 & 0.822 & 0.843 & 0.895 & 0.895 \\
\hline F4 & 0.976 & 0.826 & 0.829 & 0.864 & 0.864 \\
\hline F5 & 0.874 & 0.893 & 0.672 & 0.672 & 0.731 \\
\hline
\end{tabular}

Table 11: First order linear regression equations, rate of drug release of different formulations of Montelukast sodium press coated tablets.

\begin{tabular}{|c|c|c|}
\hline \multirow{2}{*}{ Formulation code } & Zero order & First order \\
\cline { 2 - 3 } & $\mathbf{K}_{\mathbf{0}}$ & 0.010 \\
\hline $\mathrm{X} 1$ & 0.138 & 0.034 \\
\hline $\mathrm{X} 2$ & 0.202 & 0.013 \\
\hline $\mathrm{X} 3$ & 0.224 & 0.030 \\
\hline $\mathrm{X} 4$ & 0.220 & 0.014 \\
\hline $\mathrm{X} 5$ & 0.130 & \\
\hline
\end{tabular}




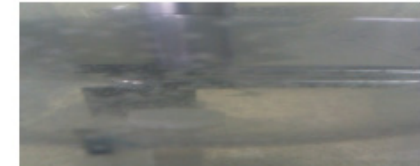

13(a) $1^{\text {st hour }}$

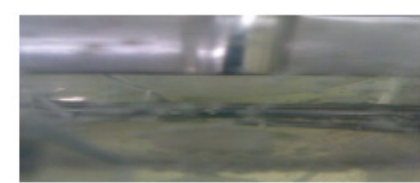

13(c) $3^{\text {nd hour }}$

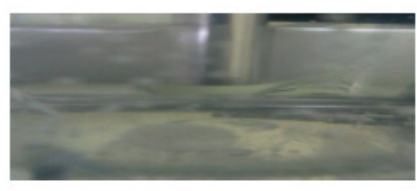

13(b) $2^{\text {nd hour }}$

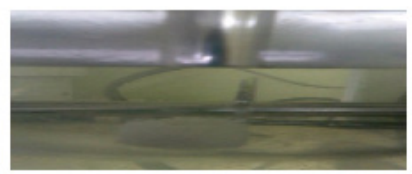

13(d) $4^{\text {to }}$ hour

Figure 8: Optimized press coated tablet showing drug release after lag time.

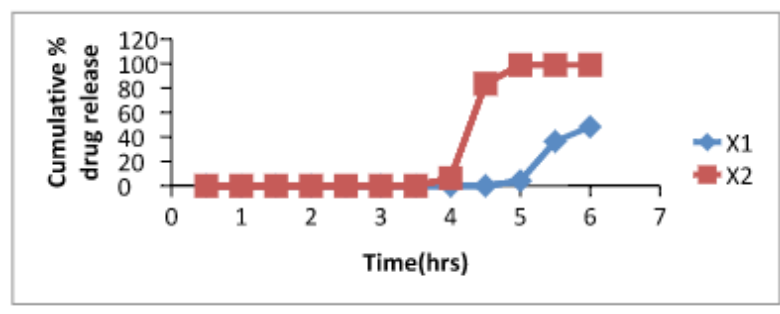

Figure 9: Plot of time vs cumulative \% drug release of $\mathrm{X} 1, \mathrm{X} 2$ formulations.

\section{Conclusion}

In the 5 trials, the optimized formulation was X2 trial which releases the Montelukast sodium immediately after a lag time. The core was compared with the pure drug where it showed $46.26 \%$ of drug release in 15 minutes whereas core exhibited $98.96 \%$ in 15 minutes.

\section{References}

1. Shangraw RF (1989) Compressed tablets by direct compression. Liebermann HA, et al. B. Pharmaceutical dosage forms, Tablets. (2 ${ }^{\text {nd }}$ edn). Marcel Dekker, New York, USA, 1: 195-243.

2. Sachin S, Neeraj K (2007) Pulsatile Drug Delivery: Current Scenario. Current Research and Information. Pharm Sci 8(2): 27-33.

3. Ravi KRJ, Veera JM, Mohamed STS, Madhu SCC (2009) Review on: Pulsatile Drug Delivery Systems. J Pharm Sci Res 1(4): 109-115.

4. Veena Belgamwar, Madhuri Gaikwad, Ganesh Patil, Sanjay Surana (2008) Pulsatile drug delivery system. Asian J Pharm 2(3): 141-145.

5. Shweta Arora, Ali J, Alka Ahuja, Sanjula Baboota, Qureshi J (2006) Review of pulsatile drug delivery systems: an approach for controlled drug delivery. Indian J Pharm Sci 68(3): 295-300.

6. Smolensky MH, Peppas NA (2007) Chronobiology, drug delivery and chronotherapeutics. Adv Drug Deli Rev 59(9-10): 828-851.

7. Jha N, Bapat S (2004) Chronobiology and chronotherapeutics.

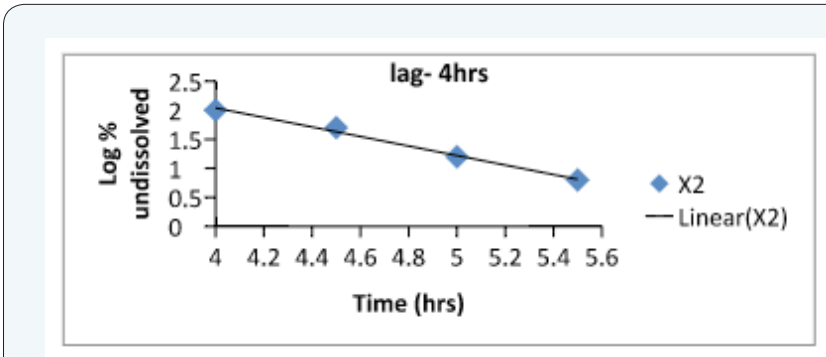

Figure 10: Plot of time vs log \% undissolved of the optimized X2 formulation.

Kathmandu Univ Med J 2(8): 384-388.

8. Patel KM, Karna N, Biswal B, Patel J (2001) Preparation and Evaluation of Pulsatile Drug Delivery System Containing Terbutaline Sulphate. International research journal of pharmacy 2(2): 113-119.

9. Young-II, Jeong (2001) Pressure controlled colon delivery capsules of Flurbiprofen. Journal of controlled release 71: 75-182.

10. Mastiholimath VS, Dandagi PM, Jain SS, Gadad AP, Kulkarni AR (2007) Time and $\mathrm{pH}$ dependent colon specific, pulsatile delivery of theophylline for nocturnal asthma. Int J Pharm 328(1): 49-56.

11. Janugade BU, Patil SS, Patil SV, Lade PD (2009) Press coated tablet of Montelukast sodium. International journal of chem Tech research 1(3): 690-691.

12. Cooper J, Windheuser J (1956) The pharmaceutics of coating tablets by compression. J Am Pharm Assoc 45(8): 542-545.

13. Sungthongjeen S, Puttipipatkhachorn S, Paeratakul O, Dashevsky A, Bodmeier R (2004) Development of pulsatile release tablets with swelling and rupturable layers. J Control Release 95(2): 147-159.

14. Ross AC, MacRae RJ, Walther M, Stevens HN (2000) Chronopharmaceutical drug delivery from a pulsatile capsule device on programmable erosion. J Pharm Pharmacol 52(8): 903-909.

15. Chaudhari SP, Chaudhari PD, Mistry CJ, Patil MJ, Barhate NS (2007) The effect of core and coating composition on drug release from directly compressed time-controlled release tablets. Pharm Tech 31: 132-144. 